\title{
Incidence of Acute and Subacute Complications Associated To Bile Duct Stents, in Patients with Advanced Cancer: A Single Center Experience.
}

\section{Alex Corbera}

Hospital del Mar https://orcid.org/0000-0003-2199-6102

\section{Alejandro Rios}

Hospital del Mar

\section{Laura Visa}

Hospital del Mar

David Casadevall

Hospital del Mar

Lucas Izarbe

Hospital del Mar

Luis Barranco

Hospital del Mar

\section{Aleksandar Radosevic}

Hospital del Mar

Sonia Servitja ( $\square$ sservitja@psmar.cat)

Hospital del Mar

\section{Research Article}

Keywords: hyperbilirubinemia, pancreatic cancer, biliary duct cancer, palliative care, biliary stent.

Posted Date: July 30th, 2021

DOl: https://doi.org/10.21203/rs.3.rs-626729/v1

License: (1) (1) This work is licensed under a Creative Commons Attribution 4.0 International License. Read Full License 


\section{Abstract \\ Background:}

Pancreatic cancer ( $\mathrm{PCa}$ ) and biliary tract carcinomas $(\mathrm{BTCa})$ have high morbidity and mortality rates. Bile duct obstruction (BDO) develops in approximately $65-75 \%$ of $\mathrm{PCa}$ at diagnosis, delaying the administration of an optimal treatment. In patients not candidates for surgery, BDO is usually treated through endoscopy-guided placement of self-expanding stents in the bile duct.

\section{Methods:}

In this retrospective study, we sought to describe clinical characteristics and outcomes of a cohort of patients with BDO of malignant origin who underwent biliary stent placement (BSP), with a special focus regarding complications developed after the procedure. Patients with $\mathrm{PCa}$ or BTCa who underwent BSP from 2014 to April 2019 at our institution were included in this cohort. Demographic and clinicopathologic characteristics were collected. Statistical analysis stratified according to ECOG performance status. Specific information regarding stent material (metallic vs. plastic), as well as incidence and type of complications derived from BSP was also recorded.

\section{Results:}

One-hundred fifteen patients were included. The median age was 72 years. Sixty-six patients $(57 \%)$ had PCa. All patients presented hyperbilirrubinemia, which decreased after the procedure in 111 (96\%) patients. Complications were observed in $44(38 \%)$ patients, most of which $33(75 \%)$ were infections. Median time to complication was one month. The mean overall survival (OS) in our study was 20.3 weeks.

\section{Conclusion:}

BSP effectively decreased hyperbilirubinemia in patients with BDO, however, the procedure associated a significant rate of infectious complications, which can further compromise an effective anti-cancer therapy as well as optimal palliative strategies.

\section{Introduction}

In the past decades, we have witnessed significant improvements in the clinical management of cancer patients, with the consequent overall improvement in cancer survivorship and quality of life ${ }^{1}$, however, in order to receive optimal treatments, patients must fit candidates. Importantly, it has become clear that the management of cancer patients requires a highly specialized multidisciplinary team that can deliver 
multimodal interventions ${ }^{2}$. Therapeutic interventions are not only necessarily intended to achieve a cure, but are also essential to the approach and management of cancer and/or treatment-related complications. If successful, these interventions may have a remarkable impact on patient survival and quality of life (QoL).

The global annual incidence of PCa and BTCa is 458,918 and 219,420 respectively, representing $2.5 \%$ and $1.2 \%$ of all newly diagnosed cancer cases ${ }^{3}$. In Spain in 2018 PCa and BTCa were responsible for 7,120 and 5,141 deaths respectively, whereas the 2020 estimates for PCa were 8,169, and gall bladder as well as BTCa $2,975 .{ }^{4}$ Despite significant advances in global cancer care and personalized medicine approaches, patients with PCa and BTCa still harbor a dismal prognosis. One of the main reasons for such prognosis is the diagnosis at advanced stages, limiting surgical treatment with curative intent.

Biliary duct obstruction (BDO) is one of the most frequent complications associated to $\mathrm{PCa}$ and $\mathrm{BTCa}$, affecting approximately $65-75 \%$ of patients at diagnosis ${ }^{5}$. Less frequently, biliary obstruction may be caused by metastases. Biliary obstruction often manifests as hyperbilirubinemia, impaired liver function, as well as severe abdominal pain, requiring local intervention before considering any other cancer-specific therapeutic approach. Even for patients not amenable for chemotherapy, management of BDO often represents a necessary step towards successful palliative treatment. In this setting, placement of a biliary stent usually represents the treatment of choice, and it is recommended for patients with a life expectancy greater than 3 months. ${ }^{6}$

The ESGE (European Society of Gastrointestinal Endoscopy) recommends the use of metallic stent in palliative drainage, as well as antibiotic prophylaxis. It is important to take into account the recommendation of a self-expandable metallic stent (SEMS) in cases where the expected survival greater than six months. ${ }^{11}$

Biliary stent placement can be performed through endoscopic retrograde cholangiopancreatography (ERCP), or transparietohepatic cholangiography (TPC) ${ }^{7}$. Both techniques involve the placement of a stent in the bile duct, in order to restore the bile flow, thus improving liver function. ${ }^{5}$ There are different types of stents depending on their fabrication material, according to the literature, metallic stent last longer than plastic ones; consequently, survival is a key factor when choosing the most adequate stent ${ }^{8,9}$. Most patients do not experience complications during the procedure ${ }^{10}$. However, secondary complications may arise in the course of days or months ${ }^{10}$. These complications are mostly infectious or stent obstructions. According to the reported literature, complications develop in $43 \%$ of all cases, of which $39 \%$ are purely infectious, $29 \%$ obstructive, and $32 \%$ combined. The risk of developing stent-related complications increases with a longer follow-up period ${ }^{10}$. These complications can limit the administration of subsequent systemic treatment.

In the present study, we reviewed a cohort of patients who underwent biliary stent placement at our institution. Our aim was to assess the nature and rate of complications, as well as their time of onset. 


\section{Material And Methods}

\section{Patients}

We performed a retrospective analysis of all patients treated for malignant biliary obstruction at the Hospital del Mar University Hospital, between April 2014 through December 2018. Included patients were $>18$ years old, affected by a solid tumor associated with a malignant BDO requiring a biliary stent patients with hepatocellular carcinoma were excluded. The follow up period began at the time of stent insertion until death or the end of study period (April 2019).

\section{Clinical data and events}

We obtained clinical data from electronic health records, including patient demographics, Eastern Cooperative Oncology Group Performance Status (ECOG PS), primary tumor site, tumor stage, indication of biliary stent (included in radical treatment, prior to palliative chemotherapy, or as a part of best supportive care), technique used (ERCP or TPC), and biliary-stent material.

The main objective was to analyze the incidence of complications related to biliary stent insertion, from the period between biliary stent insertion to first complication (TTC), regardless of the time elapsed since the insertion. The number of complications, type of complication (infectious, obstruction or migration), and overall survival (OS) were recorded for all patients.

\section{Statistical analysis}

Wilcoxon signed-rank test was used to compare bilirubin levels on admission, versus discharge values, a nonparametric distribution of the data was assumed. Survival differences between patient subgroups were estimated using a univariate log-rank test and plotted as Kaplan-Meier curves. All statistical analyses were performed with R Software version 3.6 .0 or later.

\section{Results}

Between April 2014 and December 2018, a total of 115 cancer patients were treated for BDO in our institution. Patient characteristics are described in Table 1. Median age was 72 years old (range 32-94), fifty-two patients (45.2\%) were female, $70 \%$ of the patients had an ECOG PS $\geq 2$ before the procedure. Fifty-seven percent of the patients were diagnosed with PCa, $24 \% \mathrm{BTCa}, 7 \%$ colorectal cancer, $6 \%$ gastric cancer, and $7 \%$ from other neoplasms. Forty-four percent of the patients had metastatic disease at diagnosis, and in $24 \%$ of the cases BDO was due to disease relapse or progression. In most cases (75\%), the stents were placed at the time of diagnosis of the neoplasm. The most common technique was ERCP (83.5\%), metallic stents were placed in $88 \%$ of the cases. Median follow from stent insertion to death, or end of study follow-up was 142 days (range 5-1776 days).

Prior to treatment, all cases were discussed by a multidisciplinary tumor board, 14 cases were candidates to curative treatment. Surgery was performed in 13 patients, however, only 3 patients received adjuvant 
treatment with chemotherapy, one patient received adjuvant treatment with concurrent chemoradiotherapy, and one patient received radical intent treatment with concurrent chemo-radiotherapy. In the palliative set of patients $(n=101)$, chemotherapy was administered to 39 patients, those not amenable to chemotherapy received best supportive care (62 patients).

Bilirubin at the time of admission was compared with bilirubin at discharge. The median [range] of bilirubin levels before the procedure was $12 \mathrm{mg} / \mathrm{dl}$ [0.4-39], after the stent placement, the values descended to $3 \mathrm{mg} / \mathrm{dl}[0.3-20]$ within the admission period. The median difference was $7 \mathrm{mg} / \mathrm{dl}$. Bilirubin levels decreased after stent placement in all, except four patients (Fig. 1).

Sixty-one complications were reported in 44 patients (38\%). Twenty-nine patients (66\%) developed a single complication, whereas the remaining 15 (34\%) developed multiple complications (Table 2). Regarding the tumor type, twenty-two patients (33\%) with PCa developed a complication, compared to 14 patients (52\%) with BTCa.

Infectious complications developed in $69 \%$ of the PCa patients, and $68 \%$ of the BTCa. The mean time to appearance [range] of the first complication was 22 days [1-732] after stent placement. Only 10 patients (8.6\%) developed a complication within the first 72 hours post-procedure. Patients with an ECOG PS of $2-3$, had a brifer time to complications, compared to patients with an ECOG PS $0-1$ ( $p=0.041)$ (Fig. 2).

Fifty-two patients developed an infectious complication, representing an $85 \%$ of all complications, microbiological isolation was obtained in 18 patients (41\%), E. coli and K. pneumoniae were the most common isolated microorganisms. Eight patients (18\%) presented with a radiological suggestion of obstruction or loss of stent patency, implying an infectious complication (ie. bile duct dilation). Infection was detected in 21 patients (48\%) by clinical or analytical disturbances (increased C-reactive protein, leukocytosis and/or neutrophilia, or deterioration of liver function).

Antibiotic pre-procedure prophylaxis with amoxicillin/clavulanic acid was administered to 72 patients (62\%), of which 12 (37.5\%) developed an infectious complication. Contrastingly, infectious complications occurred in 10 out of 43 patients $(41.7 \%)$ who did not receive antibiotic prophylaxis. Five patients in each group developed cholangitis during the first 72 hours after the procedure. No statistically significant differences were found regarding the use of prophylactic antibiotic between the two groups $(p=0.51)$.

In our study, the median OS was 20.3 weeks (95\% Cl 12-24) (Fig. 3). The median OS in the palliative treatment group was 12 weeks $(95 \% \mathrm{Cl} 7.6-16.7)$, whereas the group receiving a radical intention treatment was 77.6 weeks (95\% Cl 44.7-102.4) (Fig. 4), with a statistically significant difference between groups $(p<0.001)$. Mortality within thirty days after stent placement was $17 \%$ (20 patients), half of these patients (10) deceased due to disease progression, seven had an infectious complication, two developed a pancreatitis post ERCP, and one developed a myocardial infarction not related to the stent placement. It is noteworthy, that $10 \%$ of the patients received an oncologic treatment prior to the stent placement.

There was a statistically significant difference in OS according to ECOG performance status (Fig. 5). No statistically significant differences were observed regarding tumor type (data not shown). 


\section{Discussion}

In this cohort, most patients requiring a bile duct stent placement presented with either pancreatic or biliary tract neoplasms. Complications developed frequently, in thirty-eight percent of patients, most of which were infectious. Our data is in agreement with the results reported by Lamarca et $\mathrm{al}^{10}{ }^{10}$ in which $43 \%$ of the patients developed a complication, in their study infectious complications were the most frequent as well. Regarding the time from procedure to development of complications, our results differ from those of Lamarca et al, in which complications developed 4 months after the stent placement. ${ }^{10} \mathrm{~A}$ possible explanation could be related to the performance status previous to the procedure in our cohort, in which ECOG PS was $\geq 2$ for $70 \%$ of patients, whereas only $29 \%$ in the study by Lamarca.

Overall, the most frequent complication in our study, as in another reported ${ }^{10}$ was infection, most commonly caused by gram-negative rods. In agreement with other series ${ }^{10}$, cholangiocarcinoma had a higher rate of complications related to the stent. The majority of infectious complications were diagnosed based on radiological observation; microbiologic confirmation was obtained in $41 \%$ of the patients with diagnosis of infection. Most of the patients who developed an infectious complication were not receiving oncological treatment, therefore we hypothesize that infections could be related to, or as a consequence of a progressive disease, creating a suitable environment for microbiological pathogens growth.

Antibiotic prophylaxis was not associated with the development of infections, a possible explanation to this regard is that many patients were being treated for another concurrent infection, frequently cholangitis, prior to the stent placement. Furthermore, most infectious complications develop within a month after the procedure, at a moment where antibiotic prophylaxis is no longer effective.

In our study, the median overall survival was 20.3 weeks (5.1 months). Above $50 \%$ of the patients had a survival beyond six months, however $25 \%$ of the patients deceased 6.5 weeks after procedure, approximately 1.5 months. These results suggest a need for guidelines or criteria for biliary stent indications in patients in the palliative setting. The population in our study had a lower median OS, compared to one reported by Lamarca et al, of 8.6 months (approximately 34 weeks). However, the population in the study by Lamarca et al, was different, as they excluded patients who did not receive systemic treatment. Regarding mortality within a month after stent placement, it is noteworthy that $90 \%$ of the patients did not receive any oncologic treatment prior to the procedure, denoting the advanced disease patients presented with, before the stent placement, therefore suggesting a reason behind a higher mortality rate in our study.

Biliary malignant stenosis in patients with advanced/inoperable PCa or BTCa is a common complication. An effective treatment for this problem is biliary stent by ERCP or TPC, however both techniques are not absent of complications.

At our institution, a highly qualified endoscopist or radiologist performs these procedures; the most frequent method of choice is ERCP, however in the case of contraindications, such as previous surgery, the TPC approach is preferred. The ESGE recommends SEMS insertion for palliative drainage of 
extrahepatic malignant biliary obstruction, being a strong recommendation, with a high quality evidence. ${ }^{11}$ In agreement with these recommendations, $80 \%$ of the stents placed in our cohort were metallic.

To the best of our knowledge, this is one of the largest series reporting complications related to bile duct stents in an oncologic setting. A limitation of our study is the fact that it is a retrospective study, however, there is a lack of representation of this issue in the current literature. The use of stents is a feasible option, allowing a further systemic treatment for patients with impaired liver tests due to bile duct obstruction, leading to a bile drainage and further recovery of liver function. However, not all patients are amenable for a bile duct stents, pointing out the need to further improve the criteria for this procedure. Several factors are to be taken into account when analyzing the mortality within the first month post procedure, including an ECOG PS 2, infectious complications, as well as whether or not the patient receives systemic treatment. More evidence is needed elaborate multidisciplinary guidelines to improve the selection of patients who can benefit from this treatment option, further research will help clarify the aforementioned items associated to a higher mortality rate in our cohort.

\section{Declarations}

Funding: N/A

Conflicts of interest/Competing interests: N/A

Availability of data and material: April 2019

Code availability: N/A

Authors' contributions: Data collector Alex Corbera, Sonia Servitja; Manuscript writers Alex Corbera, Alejandro Rios-Hoyo, Sonia Servitja; Review datta and manuscript All the authors

Ethics approval: All the patients sing a BioBanc consent form and the approval of BioBanc is our ethical approval.

Consent to participate: all the participatns sing BioBanc consent form,

Consent for publication: N/A

\section{Conflict of interest}

The authors report no conflict of interest directly relevant to the content of this research article.

Dr. Servitja is a speaker bureau honoraria and has received travelling/attending symposium support from Roche, Pfizer, MSD; as well as a speaking or advisory honoraria from Genomichealth, Daiichi-Sankyo, Astra Zeneca, Novartis.

\section{References}


1. Ruppert, L. M., Miskovitz, G. \& Stubblefield, M. D. Cancer survivorship. Chang. Brain Impact Dly. Life 189-209 (2016). doi:10.1007/978-0-387-98188-8_9

2. Jacobson, J. O. Multidisciplinary Cancer Management: A Systems-Based Approach to Deliver Complex Care. J. Oncol. Pract. 6, 274-275 (2010).

3. Bray, F. et al. Global cancer statistics 2018: GLOBOCAN estimates of incidence and mortality worldwide for 36 cancers in 185 countries. CA. Cancer J. Clin. 68, 394-424 (2018).

4. Médica, S. española de oncología. Las Cifras del Cáncer en. Redecan, Glob. cancer Obs. 19, 1-13 (2019).

5. Krokidis, M. et al. Percutaneous palliation of pancreatic head cancer: Randomized comparison of ePTFE/FEP-covered versus uncovered nitinol biliary stents. Cardiovasc. Intervent. Radiol. 34, 352361 (2011).

6. Fukasawa, M. et al. Endoscopic biliary stenting for unresectable malignant hilar obstruction. Clin. J. Gastroenterol. 10, 485-490 (2017).

7. Ballinger, A. B., McHugh, M., Catnach, S. M., Alstead, E. M. \& Clark, M. L. Symptom relief and quality of life after stenting for malignant bile duct obstruction. Gut 35, 467-470 (1994).

8. Speer, A. G., Cotton, P. B. \& MacRae, K. D. Endoscopic management of malignant biliary obstruction: stents of 10 French gauge are preferable to stents of 8 French gauge. Gastrointest. Endosc. 34, 412417 (1988).

9. Ge, P. S. et al. Plastic biliary stent patency in patients with locally advanced pancreatic adenocarcinoma receiving downstaging chemotherapy. Gastrointest. Endosc. 81, 360-366 (2015).

10. Lamarca, A., Rigby, C., McNamara, M. G., Hubner, R. A. \& Valle, J. W. Impact of biliary stent-related events in patients diagnosed with advanced pancreatobiliary tumours receiving palliative chemotherapy. World J. Gastroenterol. 22, 6065-6075 (2016).

11. Dumonceau, J. M. et al. Endoscopic biliary stenting: Indications, choice of stents, and results: European Society of Gastrointestinal Endoscopy (ESGE) Clinical Guideline - Updated October 2017. Endoscopy 50, 910-930 (2018).

\section{Tables}

Table 1. Patient characteristics 


\begin{tabular}{|c|c|}
\hline \multicolumn{2}{|l|}{$\mathrm{N}=115$} \\
\hline \multicolumn{2}{|l|}{ Age } \\
\hline Median & $72(32-94)$ \\
\hline \multicolumn{2}{|l|}{ Gender $(n, \%)$} \\
\hline Women & $52(45)$ \\
\hline Men & $63(55)$ \\
\hline \multicolumn{2}{|c|}{ Type of Neoplasm $(n, \%)$} \\
\hline Pancreatic Cancer & $66(57)$ \\
\hline Biliary tract cancers & $27(24)$ \\
\hline Other* & $22(19)$ \\
\hline \multicolumn{2}{|l|}{ ECOG } \\
\hline 0 & $7(6)$ \\
\hline 1 & $28(24)$ \\
\hline 2 & $57(50)$ \\
\hline 3 & $23(20)$ \\
\hline \multicolumn{2}{|l|}{ Stage $\quad(n, \%)$} \\
\hline I & $1(1)$ \\
\hline II & $8(7)$ \\
\hline III & $27(24)$ \\
\hline IV & $51(44)$ \\
\hline Relapse & $28(24)$ \\
\hline \multicolumn{2}{|l|}{ Procedure $(n, \%)$} \\
\hline ERCP & $96(84)$ \\
\hline TPC & $19(16)$ \\
\hline \multicolumn{2}{|l|}{ Stent material $(n, \%)$} \\
\hline Metallic & $101(88)$ \\
\hline Plastic & $9(8)$ \\
\hline Unknown & $5(4)$ \\
\hline
\end{tabular}




\begin{aligned} No complications & $71(62) \\$\hline Any complication & $429(66) \\ 1$ complication & $4(38) \\ 2$ complications & $14(32) \\ 4$ complications & $1(2)\end{aligned}$

*Colon cancer, lung cancer, gastric cancer, ovarian

Table 2. Complications related to the stent placement

\begin{tabular}{ll} 
Complications & $\mathbf{n = 6 1}$ \\
\hline Infection & $52(85 \%)$ \\
\hline Obstruction & $7(12 \%)$ \\
\hline Migration & $2(3 \%)$
\end{tabular}

\section{Figures}

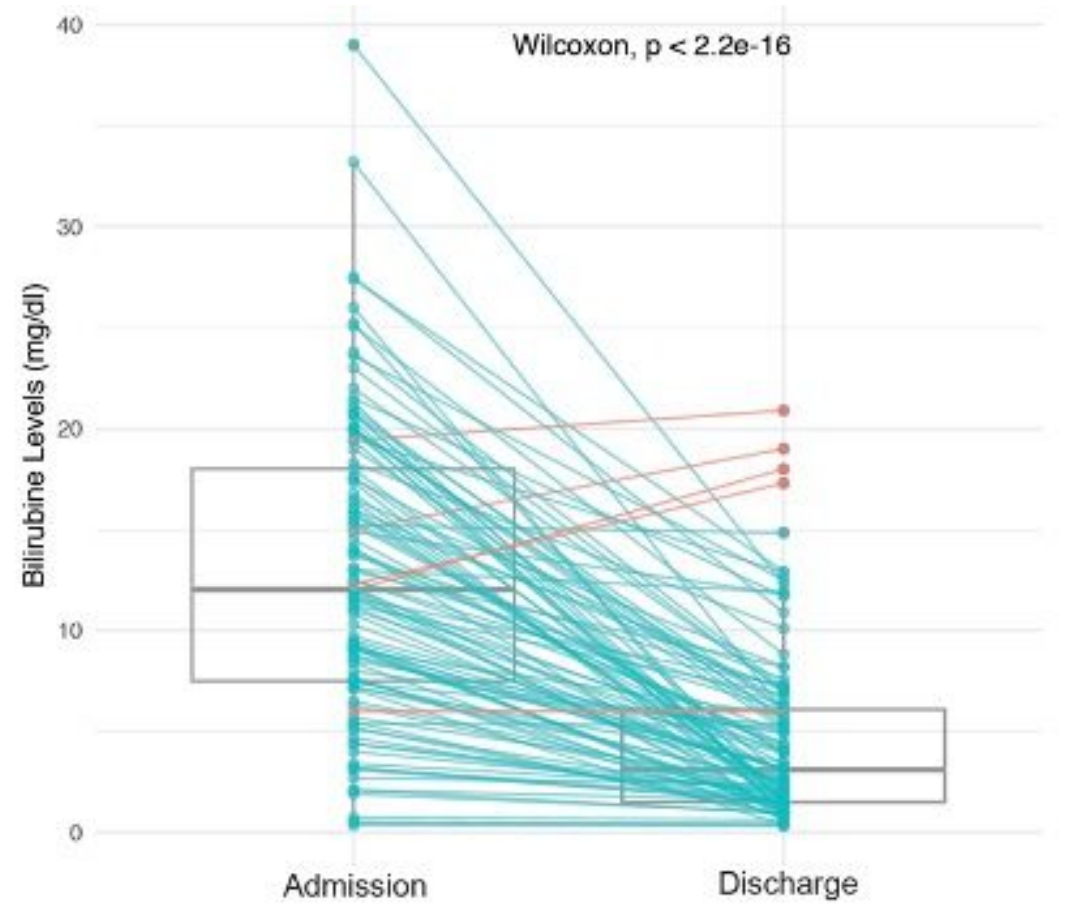

Fig 1. Differences between bilirubin levels at admission and at discharge

\section{Figure 1}

See image above for figure legend 
Time to complication by PS

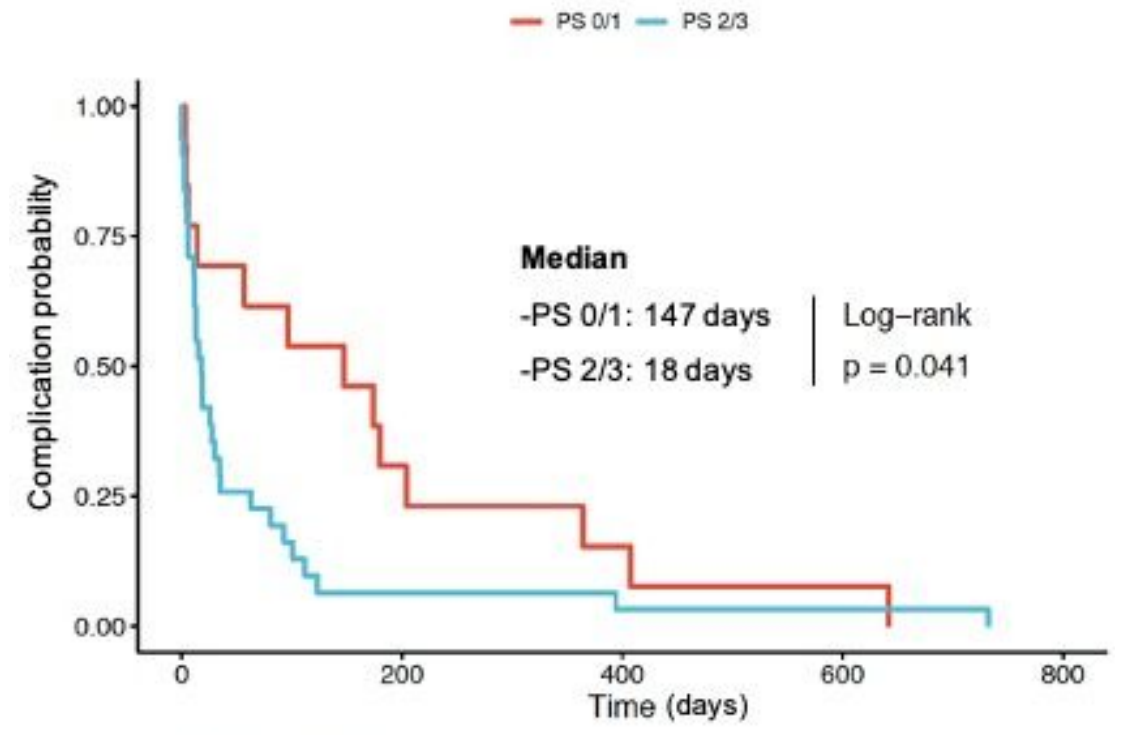

Number at risk

$\begin{array}{rllll}13 & 4 & 2 & 1 & 0 \\ 31 & 2 & 1 & 1 & 0\end{array}$

Fig 2. Time to complication, according to Performance Status

Figure 2

See image above for figure legend

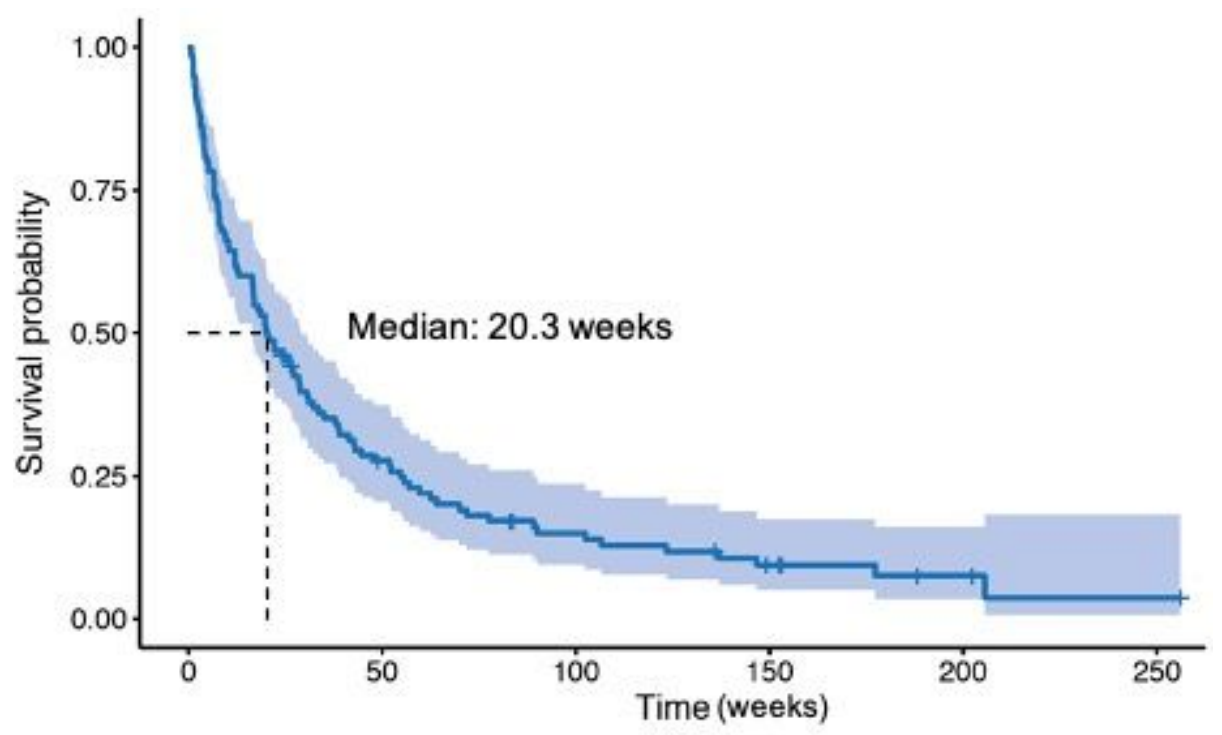

Number at risk

$\begin{array}{llllll}-115 & 29 & 14 & 7 & 3 & 1\end{array}$

Fig 3. Overall survival

\section{Figure 3}

See image above for figure legend 
Survival by treatment indication

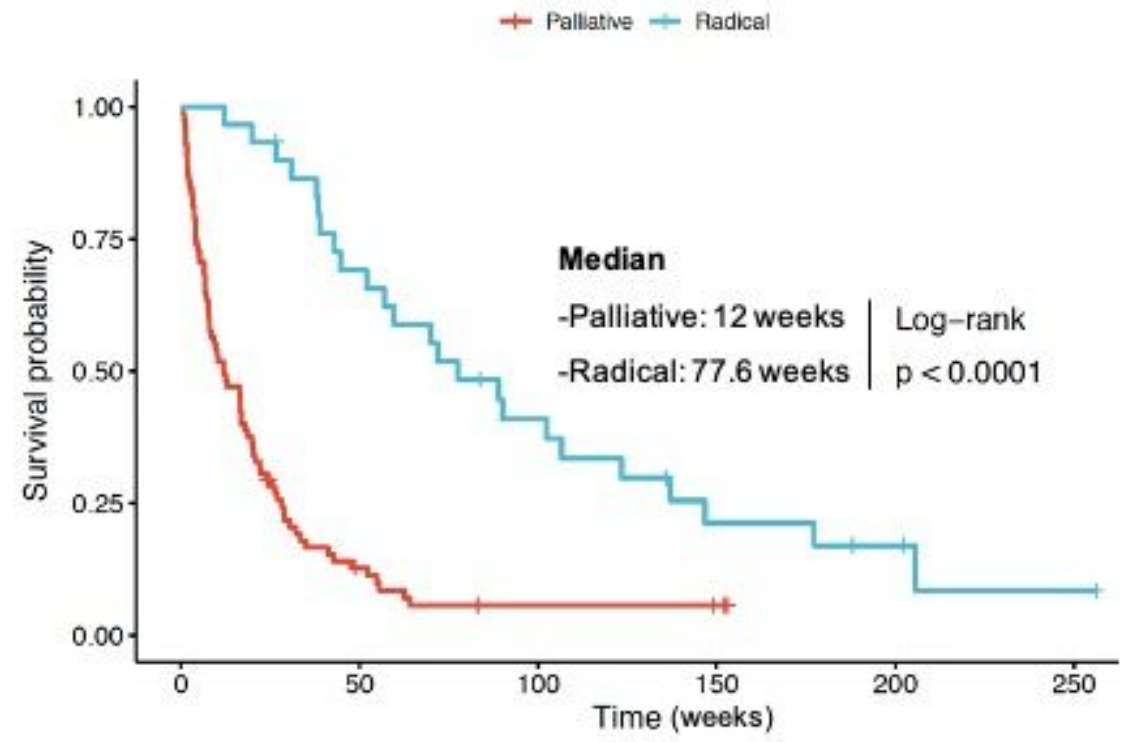

Number at risk

$\begin{array}{rrrrrr}85 & 9 & 3 & 2 & 0 & 0 \\ -30 & 20 & 11 & 5 & 3 & 1\end{array}$

Fig 4. Overall survival in patients with palliative vs radical intention

\section{Figure 4}

See image above for figure legend

Survival by PS

$$
+\mathrm{PSO} 1 \mathrm{PS} 23
$$

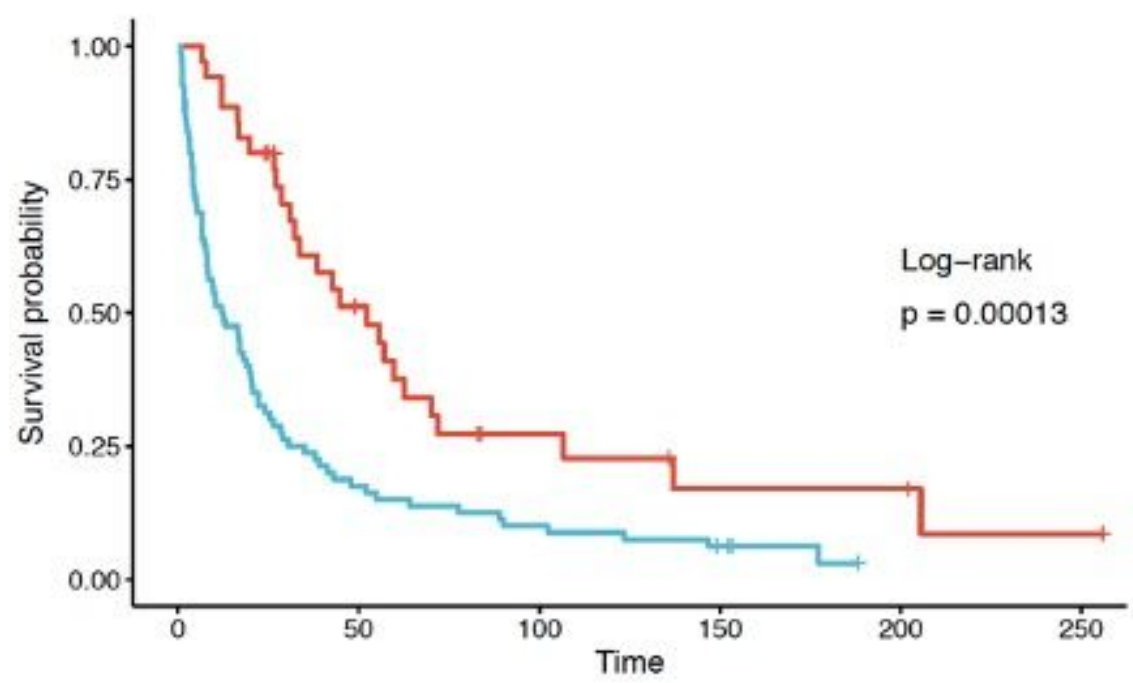

Number at risk

$\begin{array}{rrrrrr}-35 & 15 & 6 & 3 & 3 & 1 \\ -80 & 14 & 8 & 4 & 0 & 0\end{array}$

Fig 5. Overall survival in patients with PS 0-1 vs PS 2-3

Figure 5 
See image above for figure legend

Page 13/13 